\title{
Electrochemical deposition of CoNi micro/nanostructures as new materials for electrochemical sensing of glucose
}

J. Vilana, M. Lorenzo, E. Gómez, E. Vallés*

Ge'CPN, Departament Química Física, Institut de Nanociència i Nanotecnologia (IN $\left.{ }^{2} U B\right)$, Universitat de Barcelona, Martí i Franquès 1, 08028, Barcelona, Spain.

*Corresponding Author

Dr. Elisa Vallés, e.valles@ub.edu, Fax: +34 934021231

\begin{abstract}
CoNi micro/nanostructures (films and nanowires of different properties) have been synthesised, through a facile and low cost method, as catalysts for glucose electro-oxidation, being promising material for non-enzymatic glucose sensors. The synthesis is a one-step procedure able to prepare, from a single solution, films or nanorods with different composition, shape and morphology. The electrocatalytic activity of the CoNi films and glassy carbon/CoNi nanorods for the glucose oxidation in basic medium has been investigated. The glucose oxidation current is clearly detected over the oxidized alloy. Voltamperometry and chronoamperometry techniques allow sensing the glucose over the CoNi structures prepared. The best catalysts are the films synthesised at $-1.0 \mathrm{~V}$ and the nanorods at $-0.8 \mathrm{~V}$, which moreover minimizes the amount of sensing material.
\end{abstract}

Keywords: electrochemical synthesis, CoNi catalysts, nanorods, glucose oxidation, sensing

\section{Introduction}

The fast development during last decades of nanostructured materials has generated a growing interest for new technologic applications. The nanomaterials have been successfully applied in fields as electronics, energy sources, information storage or medicine. In the last years an increasing interest in investigating their use as electrochemical electrodes for sensor devices has been detected. The attention has especially focused in sensing molecules of biological interest [1-3]. For nanostructures synthesis, the facile and non-expensive electrochemical method can play a significant role.

In the present work we propose the use of micro/nano electrosynthesis for the preparation of CoNi catalysts for glucose electro-oxidation, as potential materials in electrochemical nonenzymatic glucose sensors. Glucose is one the biomolecules of greater importance in clinical 
diagnosis and food and pharmaceutical industries, being the development of new glucose sensors the aim of recent investigations [4-7]. Metallic nanostructures have been tested to replace enzymatic biosensors, to improve stability and selectivity $[2,8,9]$. Glucose sensing electrodes with noble metals have been proposed [10], but recent publications have been focused in the use of nanostructures from other cheaper metals as nickel [1,5,7-9].

A few recent investigations describe the use of $\mathrm{Ni}$ bimetallic nanostructures for glucose sensing $[4,6,11]$, some of them CoNi nanoparticles, also tested for sensing other biomolecules such as uric acid [3]. The current work presents an easy electrochemical way of synthesis of CoNi nano and micro structures. By first time the sensing capability of CoNi nanowires is tested and the behaviour of CoNi films of similar composition but different morphology is compared. The application of different synthesis conditions, from a single bath, will permit to obtain $\mathrm{CoNi}$ catalysts with different properties (composition, roughness, shape), and the best ones for glucose oxidation could be selected for non-enzymatic sensors.

\section{Experimental}

CoNi deposits of different shapes (films and nanorods) have been prepared by potentiostatic electrodeposition, at $21^{\circ} \mathrm{C}$ in an electrolytic deoxygenated bath at $\mathrm{pH} 3$ containing $\mathrm{CoCl}_{2} 0,2 \mathrm{M}$, $\mathrm{NiCl}_{2}, 0,9 \mathrm{M}$ and $\mathrm{H}_{3} \mathrm{BO}_{3}$ 0,5 $\mathrm{M}$ in Millipore Milli $\mathrm{Q}$ water. A platinum spiral was used as auxiliary electrode and an $\mathrm{Ag} / \mathrm{AgCl} / \mathrm{KCl} 3 \mathrm{M}$ as reference electrode. For the CoNi films preparation, flat silicon pieces with $\mathrm{Ti}(50 \mathrm{~nm}) / \mathrm{Ni}(100 \mathrm{~nm})$ seed layers were used as working electrode. For the CoNi nanorods synthesis, polycarbonate membranes ( $20 \mu \mathrm{m}$ thick, $100 \mathrm{~nm}$ pore diameter) with a sputtered gold layer ( $50 \mathrm{~nm}$ ), were used as working electrode. Once the nanorods were synthesised, the gold layer was etched with a saturated $\mathrm{KI}_{3}$ solution, the polycarbonate membrane was dissolved with chloroform until total removing and, finally, the nanowires were dried.

The electrochemical experiments (material preparation and glucose oxidation) were performed with Potentiostat/Galvanostat Autolab PGSTAT30 and GPES software.

CoNi films and nanorods composition was determined by X-rays fluorescence (XRF) with a Fisherscope XDAL X-ray Fluorescence system. The deposits were observed by Scanning Electron Microscopy (SEM) by using JSM-7100F Analytical Field Emission equipment. Thickness and roughness of the CoNi films were analyzed by a Leica DCM 3D confocal. 
The electro-oxidation test of glucose was performed in a $0.1 \mathrm{M} \mathrm{NaOH}$ solution, by using the synthesised CoNi deposits or the prepared nanorods as working electrodes. In the case of the nanorods, they were dispersed in ethanol absolute and deposited on a glassy carbon electrode ( $2 \mathrm{~mm}$ of diameter).

\section{Results and discussion}

The potentiostatic deposition on $\mathrm{Si} / \mathrm{Ti} / \mathrm{Au}$ pieces led to compact CoNi films with composition, morphology and roughness modulated by the deposition potential (Figure 1, as-deposited films, Table 1). Pyramidal grains and high roughness was observed for the films prepared the less negative potential, which have the highest cobalt percentage. Smother surfaces and lower Co percentages were obtained by decreasing the synthesis potential.

\begin{tabular}{|c|c|c|c|c|c|c|c|}
\hline \multicolumn{8}{|c|}{ Table 1} \\
\hline \multicolumn{5}{|c|}{ Characteristics of the CoNi catalysts synthesised } & \multicolumn{3}{|c|}{ Sensing properties for glucose oxidation } \\
\hline & & $\begin{array}{c}\text { Synthesis } \\
\text { potential } \\
\text { / V }\end{array}$ & $\begin{array}{l}\text { Roughness } \\
\qquad / \mu \mathrm{m}\end{array}$ & $\begin{array}{l}\text { Wt. } \\
\% \text { Co }\end{array}$ & $\begin{array}{l}\text { Sensing } \\
\text { technique }\end{array}$ & $\begin{array}{l}\text { Sensitivity / } \\
\mu \mathrm{A} \mathrm{mM}^{-1} \mathrm{~cm}^{-2}\end{array}$ & $\begin{array}{l}\text { Linear range } \\
\text { of } \\
\text { [glucose]/mM }\end{array}$ \\
\hline \multirow{6}{*}{$\begin{array}{l}\text { CoNi films } \\
\text { Geometrical area : } \\
0.25 \mathrm{~cm}^{2} \\
\text { Thickness: } 1.3 \mu \mathrm{m}\end{array}$} & \multirow{2}{*}{ A } & \multirow{2}{*}{-0.8} & \multirow{2}{*}{0.11} & \multirow{2}{*}{70} & CV & 282 & $1.5-14$ \\
\hline & & & & & CA & 353 & $0.1-1.5$ \\
\hline & \multirow{2}{*}{ B } & \multirow{2}{*}{-1.0} & \multirow{2}{*}{0.02} & \multirow{2}{*}{42} & $\mathrm{CV}$ & 298 & $1.5-14$ \\
\hline & & & & & CA & 595 & $0.1-1.5$ \\
\hline & \multirow{2}{*}{ C } & \multirow{2}{*}{-1.2} & \multirow{2}{*}{0.01} & \multirow{2}{*}{30} & CV & 303 & $1.5-14$ \\
\hline & & & & & CA & 571 & $0.1-1.5$ \\
\hline \multirow{4}{*}{$\begin{array}{l}\text { CoNi nanorods } \\
\text { Length: } 3 \mu \mathrm{m} \\
\text { Diameter: } 120 \mathrm{~nm}\end{array}$} & \multirow[b]{2}{*}{ A } & \multirow{2}{*}{-0.8} & \multirow{2}{*}{$\mathrm{n} / \mathrm{a}$} & \multirow{2}{*}{70} & $\mathrm{CV}$ & 305 & $1-10$ \\
\hline & & & & & CA & 544 & $0.1-1$ \\
\hline & \multirow{2}{*}{ B } & \multirow{2}{*}{-1.0} & \multirow{2}{*}{$\mathrm{n} / \mathrm{a}$} & \multirow{2}{*}{60} & $\mathrm{CV}$ & 243 & $1-10$ \\
\hline & & & & & $C A$ & 383 & $0.1-1$ \\
\hline
\end{tabular}

To investigate the electrochemical performance of the $\mathrm{Si} / \mathrm{Ti} / \mathrm{Ni} / \mathrm{CoNi}$ structures, cyclic voltammetry in $\mathrm{NaOH} 0.1 \mathrm{M}$ was employed, because some authors proposed that in the case of nickel, $\mathrm{Ni}(\mathrm{III})$ oxides participates in the mechanism of glucose electro-oxidation [1]. Oxidation/reduction peaks were observed (Figure 1, voltammetric profiles) that can be assigned to the $M(I I I) / M(I I)$ redox couple [12], in a similar way that for pure-nickel structures 
$[1,9]$. From 10 consecutive cycles, stationary voltammetric profile was already attained, which demonstrates that the deposits are very stable after the cycling process. As can be observed in the voltammetric curves, the more negative synthesis potentials favour the oxidation process. As the process is not totally reversible, the films surface is clearly oxidized after successive voltammetric cycles, as can be observed in the SEM pictures after the activation (Figure 1, activated catalysts).
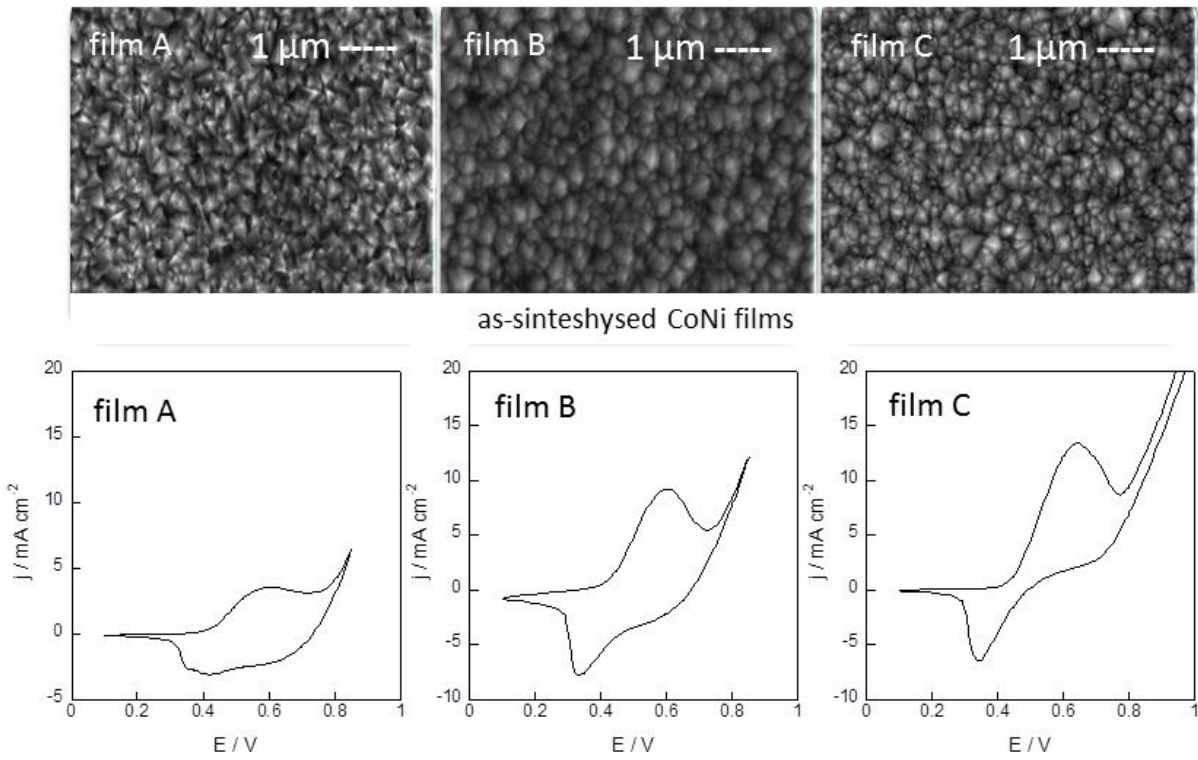

as-sinteshysed CoNi films
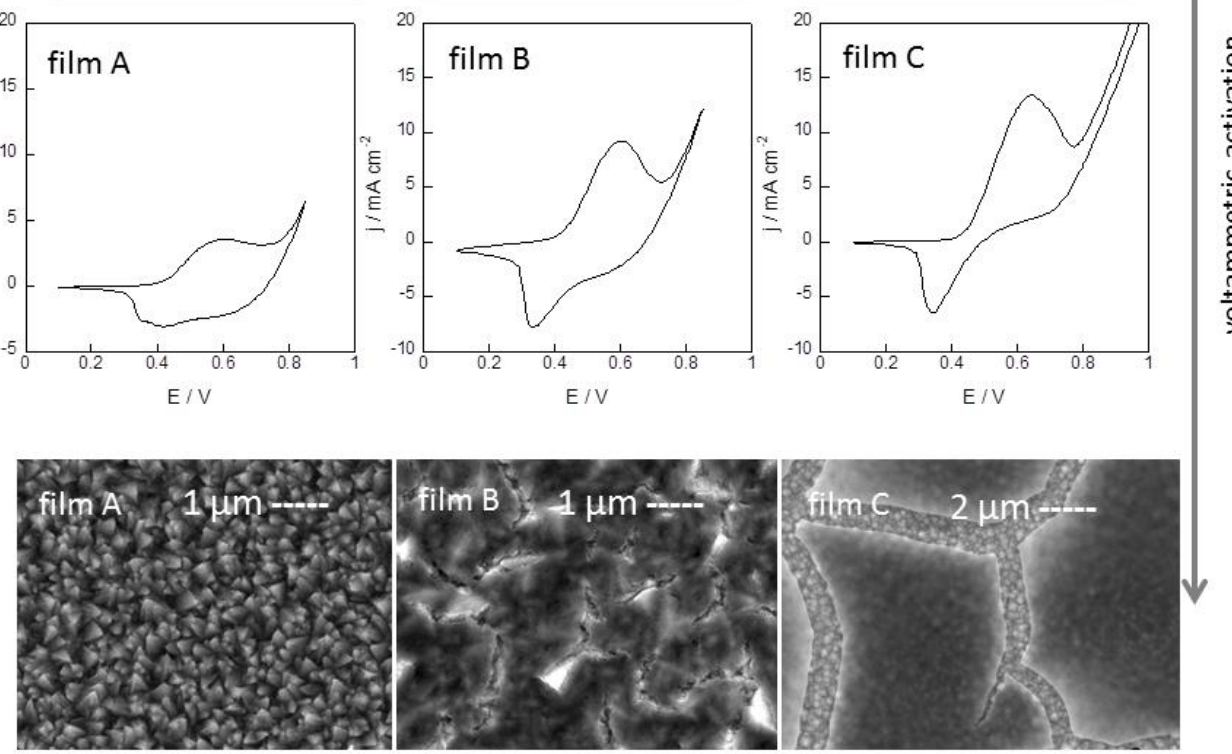

activated CoNi catalysts

Figure 1

After the activation of the films, glucose oxidation over these substrates was tested. Figure 2 shows the voltammetric profiles (CV) in a $\mathrm{NaOH} 0.1 \mathrm{M}$ solution containing different glucose concentrations; defined oxidation peaks, diffusion-controlled, depending on the glucose concentration, demonstrates the catalytic effect of the oxidized CoNi films. Oxidation current was not observed on glassy carbon electrodes and very low current was detected on the $\mathrm{Si} / \mathrm{Ti} / \mathrm{Ni}$ substrate. A linear dependence of the maximum of the peak with the glucose concentration was observed in the range tested (Figure 2). 
Also, chronoamperometric (CA) experiments were performed in which, in stirring conditions, glucose is gradually added to the solution. Potentials in the $0.5-0.65 \mathrm{~V}$ range were selected to record the current density (j), which gradually increases with glucose additions (Figure 2). Also in these conditions, linear $\mathrm{j}$-[glucose] dependence was observed. This makes the CoNi films promising structures to detect and quantify the glucose. From the two techniques (CV and CA) better results (high $\mathrm{j}$-[glucose] slope) were obtained with the CoNi catalysts synthesised at more negative potentials $(-1.0,-1.2 \mathrm{~V}$ ) (Table 1 ), probably as a consequence of the easier oxidation of the film, as can be observed in the SEM pictures after the activation.
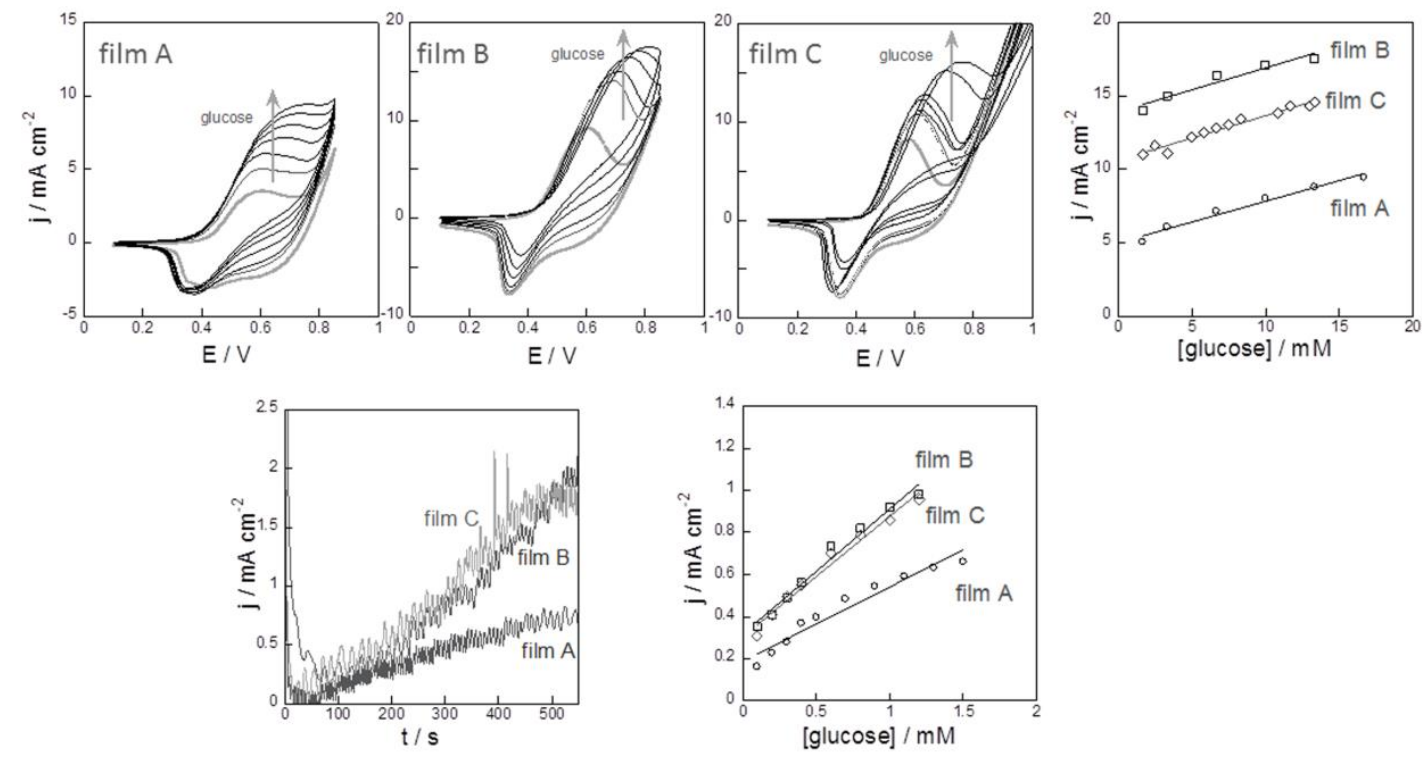

Figure 2

Similar experiments were performed with the CoNi synthesised nanorods at two potentials (characteristics in Table 1). Figure 3 shows the SEM pictures of some nanorods, as-prepared and after the activation in $\mathrm{NaOH}$; also, the $\mathrm{CV}$ curves corresponding to the glucose detection and the j-[glucose] graphics obtained by CV (upper graphic) or CA (lower graphic) experiments. The current density was obtained taking into account the calculated area of the nanorods present on the glassy carbon surface. As can be seen in the SEM images, the activation of the nanorods induces an increase of the superficial roughness, as a consequence of the surface oxidation. Linear dependence between the glucose addition and the current density was obtained in the two range of glucose concentrations tested. The sensibility is similar than in the case of CoNi films (Table 1) 

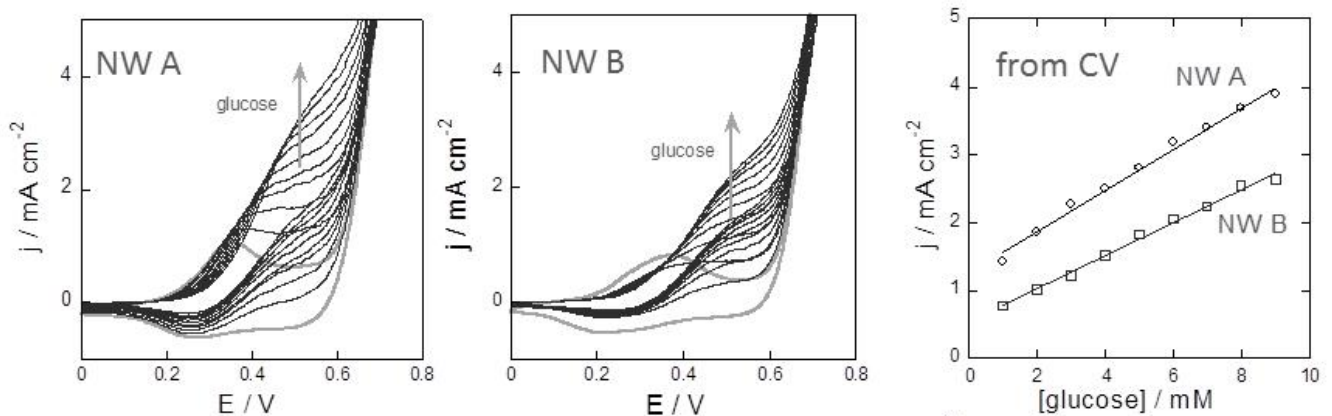

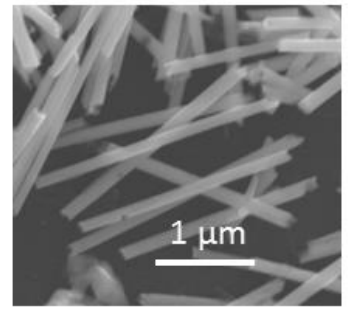

before cycling

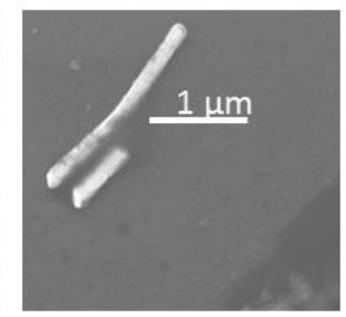

after cycling

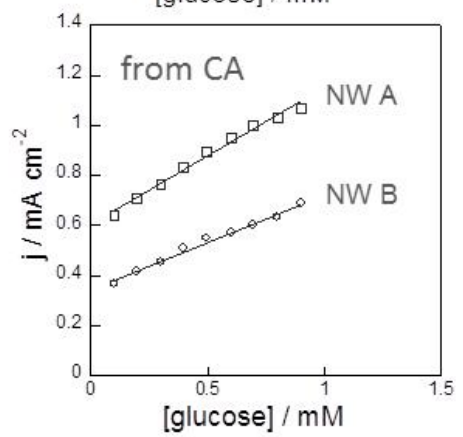

Figure 3

\section{Conclusions}

CoNi films and nanorods of different composition, activated in basic medium, have been demonstrated as catalysts for glucose electro-oxidation. The two types of materials for sensing have been obtained by means a facile one-step electrochemical synthesis procedure. Linear dependence between the oxidation current density and the glucose concentration was obtained in the two ranges of concentrations tested, lower in the chronoamperometric experiments and higher in the voltammetric ones. Therefore, the prepared structures are promising substrates for amperometric detection of glucose in non-enzymatic sensors. The better results have been obtained for the lower range of glucose concentrations and chronoamperometry technique, by using CoNi films obtained at $-1.0 \mathrm{~V}$ or CoNi nanorods prepared at $-0.8 \mathrm{~V}$.

\section{Acknowledgements}

The authors wish to thank the Centres Científics i Tecnològics de la Universitat de Barcelona for the use of their equipment and the Instituto de Microelectrónica de Barcelona (CNM-CSIC) for the $\mathrm{Si} / \mathrm{Ti} / \mathrm{Au}$ substrates supply and the metallization of the polycarbonate membranes. 


\section{References}

[1] Safavi A, Maleki N, Farjami E. Fabrication of a glucose sensor based on a novel nanocomposite electrode. Biosens Bioelectron. 2009;24(6):1655-1660.

[2] Toghill KE, Compton RG. Electrochemical non-enzymatic glucose sensors: A perspective and an evaluation. Int J Electrochem Sci. 2010;5(9):1246-1301.

[3] Singh B, Laffir F, Dickinson C, McCormac T, Dempsey E. Carbon supported cobalt and nickel based nanomaterials for direct uric acid determination. Electroanalysis. 2011;23(1):79-89.

[4] Li M, Liu L, Xiong Y, et al. Bimetallic MCo ( $M=C u, F e, N i$, and $M n)$ nanoparticles dopedcarbon nanofibers synthetized by electrospinning for nonenzymatic glucose detection. Sensors Actuators B Chem. 2015;207:614-622.

[5] Choi T, Kim SH, Lee CW, et al. Synthesis of carbon nanotube-nickel nanocomposites using atomic layer deposition for high-performance non-enzymatic glucose sensing. Biosens Bioelectron. 2014;63C:325-330.

[6] Hussain M, Ibupoto ZH, Abbasi MA, Liu X, Nur O, Willander M. Synthesis of three dimensional nickel cobalt oxide nanoneedles on nickel foam, their characterization and glucose sensing application. Sensors (Switzerland). 2014;14(3):5415-5425.

[7] Ci S, Huang T, Wen Z, et al. Nickel oxide hollow microsphere for non-enzyme glucose detection. Biosens Bioelectron. 2014;54:251-257.

[8] Liu Y, Teng H, Hou H, You T. Nonenzymatic glucose sensor based on renewable electrospun Ni nanoparticle-loaded carbon nanofiber paste electrode. Biosens Bioelectron. 2009;24(11):3329-3334.

[9] Lu LM, Zhang L, Qu FL, et al. A nano-Ni based ultrasensitive nonenzymatic electrochemical sensor for glucose: Enhancing sensitivity through a nanowire array strategy. Biosens Bioelectron. 2009;25(1):218-223.

[10] Pasta M, La Mantia F, Cui Y. Mechanism of glucose electrochemical oxidation on gold surface. Electrochim Acta. 2010;55(20):5561-5568.

[11] Liu Y, Zhang Y, Wang T, Qin P, Guo Q, Pang H. Mesoporous Ni 0.3 Co $2.7 \mathrm{O} 4$ hierarchical structures for effective non-enzymatic glucose detection. RSC Adv. 2014;4(63):33514.

[12] Tarrús X, Montiel M, Vallés E, Gómez E. Electrocatalytic oxidation of methanol on CoNi electrodeposited materials. Int . J. Hydrogen Energ. 2014;39(12):6705-6713.

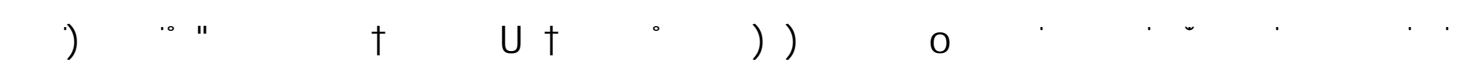

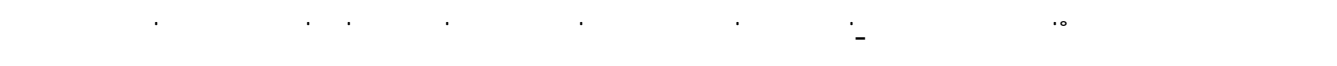


Table 1. Characteristics of the CoNi structures synthesised and their behaviour for glucose oxidation. CV: cyclic voltammetry, CA: chronoamperometry

Figure 1. SEM pictures of as-deposited CoNi films obtained at different potentials, stationary cyclic voltammograms of the films in $\mathrm{NaOH} 0.1 \mathrm{M}$ at $50 \mathrm{mV} \mathrm{s}^{-1}$, and SEM pictures of the films after the voltammetric activation

Figure 2. Upper part: Cyclic voltammograms, at $50 \mathrm{mV} \mathrm{s}^{-1}$ in $\mathrm{NaOH} 0.1 \mathrm{M}$, of the activated CoNi films with glucose addition (grey curve: $0 \mathrm{M}$ glucose) and the corresponding $\mathrm{j}$-[glucose] dependence. Lower part: Chronoamperometries by adding the glucose and the corresponding j-[glucose] dependence

Figure 3. SEM pictures of the nanorods before and after the cycling in $\mathrm{NaOH} 0.1 \mathrm{M}$. Cyclic voltammograms (CV), at $50 \mathrm{mV} \mathrm{s}^{-1}$ in $\mathrm{NaOH} 0.1 \mathrm{M}$, of the activated CoNi nanowires with glucose addition (grey curve: $0 \mathrm{M}$ glucose) and the corresponding $\mathrm{j}$-[glucose] dependence from CV (upper graphic) and chronoamperometry (CA) (lower graphic) 